\title{
Impact of the Economic Crisis on Climate Change
}

\begin{abstract}
Time flies when you're having fun. And it stands still when disaster befalls. It seems like a long time ago, but it is only about 8 months since the collapse of Lehman Brothers on 15 September 2008 triggered a global recession. Before Lehman, most assumed that economic growth would continue, with the main questions focused on its magnitude and variegation across regions and countries. The big question in environmental economics was whether and to what extent we could create and implement policy interventions that would finally break the link between economic growth and rising greenhouse gas emissions. The European Union Emissions Trading Scheme (EU ETS) has been our "big idea" in Europe to create a price for carbon that symbolises the scarcity value of the atmosphere as an absorber of greenhouse gas, and provides a market incentive to break this link.
\end{abstract}

But we are now in the land of a paradigm shift, where deep recession is suddenly upon us, compounded by constipation in credit markets generated by a banking system that at times seems to be wounded beyond repair. What is the import of this for greenhouse gas emissions in the short and medium term? The answer is that we don't know. We do know that behaviour is asymmetric - we respond differently to the risk of loss compared to gain, to decline compared to growth. But all of our current models and the associated coefficients and calibrations derive from a history of growth; only in Japan in the modern era can we empirically investigate the implications of stasis and economic decline. Also, the topography of the financial crisis is unique, and the implications unknown - we have no idea to what extent and how quickly the situation will turn around, or what will be the implications for lending and therefore for investment and consumption of the new regulatory regimes that are now taking shape.

But let's interrogate the tea leaves and see what we can see. In the EU, there will certainly be a sharp absolute fall in greenhouse gas emissions in the 2009-10 period, and probably thereafter. How sharp and how sustained will depend on what happens to economic growth, and how effective the policies are that we have put and are putting in place to break the link between growth in economy and emissions. We know that emissions in the EU ETS in 2008 fell by $3.07 \%$ compared with 2007, in spite of GDP growth in 2008 of $0.8 \%$. But in the March 08 to March 09 period, industrial production declined by $18.8 \%$ and retail sales fell by 3.1 per cent, with most of the decline occurring in the last quarter of 2008 and the first quarter 2009. This unprecedented fall in economic activity will put dramatic downward pressure on emissions, and this pressure will be accentuated by the presence of a carbon price signal which provides an incentive every day to firms in EU ETS to reduce emissions, and there are parallel incentives to increase the use of renewables and improve energy efficiency. Eurostat projects a GDP decline for the EU27 in 2009 of $-4.0 \%$ and decline again in 2010 of $-0.1 \%$. My guess is that over the next four years (2009-2012) the combination of climate change policy - and notably the carbon price - and economic decline will reduce emissions in the EU by up to $20 \%$ from 2008 levels. This means that we could meet our emissions reduction obligation in EU ETS (-21\% to a 2005 base) 8 years early. The challenge will be to ensure that the policy instruments we have put in place - the price signal from the EU ETS, incentives supporting renewable energies, and more broadly the legally binding national caps on the non-trading sectors - all are sufficient to maintain and intensify the break between economic growth and greenhouse gas emissions as we re-discover economic growth.

So the sharp decline in emissions we will experience need not be a "blip" but a precursor of sustained reduction. 
But of course this downward trajectory will only be sustained if the levels of investment required to make the transition are provided, and if the innovation that is essential is realised. And here we peer into the bowl of spaghetti that is the global financial system and wonder when and how it will cohere again into a functioning whole. The investment needs are in two broad categories - that required to finance "conventional" investment (including established renewables such as wind power) and that required to support innovation, including research, development and demonstration, venture capital, and finally mainstreaming. The amounts needed are very large. The International Energy Agency (Energy Outlook 2008) estimates that $€ 1738$ billion - 144 for transmission, 436 for distribution, and 1158 for power generation - are needed between now and 2030 by the electricity sector in OECD Europe alone to meet needs. The investment needs of innovation are likewise very substantial. As the gridlock that is inhibiting the provision of loans relaxes, investment is likely to flow mainly to conventional "safe" homes with reliable returns and little risk. If we ensure that electric utilities are allowed to pass through the cost of allowances in the electricity price, the industry should be a favoured client for such finance. It will require direct policy intervention to ensure that the capital needed to get us across the bridge to a zero carbon economy is provided in sufficient quantity and in a manner that supports and sustains excellence in innovation, including taking substantial risks in betting on the rich and confusing array of what are by definition relatively unproven technologies. I don't know what shape such policies should take, but an explicit earmarking for innovation of some of the revenues that will be generated by the auctioning of allowances from 2013 will be essential. The policy package now in place for Carbon Capture and Storage - earmarking of the revenues of 300 million allowances, allowing exemption from holding allowances for carbon that is sequestered - and the interest of some major utilities - is likely to attract the finance needed to progress.

The various Green New Deal packages that are being mooted could be an important mechanism for making up the investment gap in regard especially to innovation. And this applies to both adaptation and mitigation. As regards the former, we need to reconfigure most of our investment programmes to take account of the climate change that is now inevitable, and a Green New Deal programme can help achieve such reconfiguration.

One of the many benefits of the EU ETS is that it does provide reliable emissions for the most recent year (2008); but a limitation is that we have to wait until March 2010 for the next wave of data.

It would improve our ability to make connections, improve our analyses and specifically track whether we are indeed breaking the link between GDP and emissions if we had quarterly data on emissions in the EU ETS to mirror the excellent quarterly data on GDP provided by Eurostat.

So the inevitability of sharp falls in greenhouse gas emissions engendered in part by the economic crisis can become a permanent gain if we maintain our carbon pricing policies via EU ETS and other related policies, and ensure that funds are freed up and preferentially allocated to support investment both for adaptation to climate change, and innovation in both adaptation and mitigation. Auction revenues from EU ETS from 2013 are the most likely source, although Green New Deal initiatives happening independently of auctioning of allowances should also be designed to help.

For the European policy system, it is important that the crisis be seen as an opportunity to make big reductions that we can then lock in, and ensure that carbon price, investment and other policies nudge us all toward innovations that in turn give us the tools to be a low carbon society, with a business model that combines prosperity with responsibility, and provides an example worth emulation to the rest of the world.

Frank J. Convery,

Heritage Trust Professor of Environmental Policy, University College Dublin, Ireland. 